\title{
El Plano Cartesiano en estudiantes de Quinto Básico: su Resignificación en una Situación Específica
}

\author{
The Cartesian Plane in Fifth Grade students: its Re-signification on a \\ Specific Situation
}

\author{
Angélica Aravena* \\ ORCID iD 0000-0002-5006-5510 \\ Astrid Morales** \\ ORCID iD 0000-0001-7569-6362
}

\begin{abstract}
Resumen
Se presentan los resultados de una investigación relativa a la resignificación del plano cartesiano en un grupo de estudiantes de quinto básico de una escuela chilena. El discurso matemático escolar correspondiente carece de situaciones de enseñanza significativas para introducir y comprender el uso de ese plano, más bien fomenta una matemática prescrita, donde no es necesaria su construcción. Desde un enfoque cualitativo, a través de la indagación del proceso de construcción de un conocimiento matemático, la resignificación se evidencia a través del diseño de una situación específica que promueve la necesidad de la construcción del plano cartesiano. En los argumentos y discusión sostenidos por el grupo de estudiantes se evidenció la construcción de ejes ortogonales, las nociones de distancia, origen y ubicación de puntos considerando coordenadas rectangulares.
\end{abstract}

Palabras Claves: Plano Cartesiano. Resignificación. Socioepistemología. Sistema de Referencia.

\begin{abstract}
We report the results of a research on the re-signification of the Cartesian plane in a group of fifth-grade students of a Chilean school. The corresponding mathematical school discourse lacks meaningful teaching situations to introduce and to understand the use of that plane, and rather fosters a prescribed mathematics, that you do not need to build. That re-signification was evidenced through the design of a specific situation promoting the need of building the Cartesian plane. In the arguments and discussion held, the group evidenced building orthogonal axes, and the notions of distance, origin, and location of points considering rectangular coordinates
\end{abstract}

Keywords: Cartesian Plane. Re-signification. Socioepistemology. Reference System.

\section{Introducción}

\footnotetext{
* Magister en Didáctica de la Matemática por la Universidad Católica de Valparaíso (PUCV). Profesora en Didáctica de la Matemática de la Escuela de Educación de la Universidad Mayor (UM), Santiago, Chile. Dirección postal: Avenida Manuel Montt, 318, Providencia, Santiago, Región Metropolitana, Chile, C.P: 7500628. E-mail: angelica.aravena@mayor.cl.

** Doctora en Matemática Educativa por el Instituto Politécnico Nacional Centro de Investigación en Ciencia Aplicada y Tecnología Avanzada (CINVESTAV). Profesora en Didáctica de la Matemática del Instituto de Matemática de la Pontificia Universidad Católica de Valparaíso (PUCV), Valparaíso, Chile. Dirección Postal: Blanco Viel, 596, Cerro Barón, Región de Valparaíso, Chile, C.P: 2340000. E-mail: astrid.morales@pucv.cl.
} 
El plano cartesiano se presenta transversalmente en cada uno de los ejes temáticos de las actuales Bases Curriculares formuladas por el Ministerio de Educación Chileno, (MINEDUC, 2012) ya sea como sistema de referencia que favorece la ubicación y localización espacial, o como una herramienta que permite la comprensión de otros contenidos matemáticos, tales como funciones, isometrías, lectura de gráficos etc.

A pesar de su uso reiterado, en nuestra práctica pedagógica observamos, permanentemente, acciones que reflejan los problemas que tienen los estudiantes en la comprensión y uso de los ejes cartesianos, como por ejemplo, la ubicación de puntos y figuras geométricas en una determinada posición del plano. Compartimos con Saiz (2003) que hay comportamientos de los sujetos, en el cotidiano, que muestran las dificultades que experimentan para orientarse en un ambiente nuevo y para utilizar o elaborar planos y mapas.

Acuña (2001), evidencia los problemas que tienen estudiantes de secundaria para establecer el orden de las coordenadas de los puntos en el plano cartesiano pues, en lugar de utilizar ese plano para trabajar en un espacio orientado de dos dimensiones, limitan su uso solo a la descripción de parejas de números negativos o positivos.

De acuerdo a esta problemática, observamos en las Bases Curriculares Chilenas (MINEDUC, 2012) y en los textos de estudio (ASKEY, 2014) entregados por el MINEDUC, un indicio de lo que provocaría esta dificultad en los estudiantes: la presencia de una caracterización del plano cartesiano, en cuanto a su forma y descripción, más no en su uso y funcionalidad.

Para comprender y hacer propuestas que permitan construir aprendizajes escolares, la Socioepistemología propone marcos de referencia que favorecen una matemática funcional $^{1}$ en situaciones específicas, resignificando ${ }^{2}$ el conocimiento matemático puesto en juego, es decir, usando y otorgándole significado a ese conocimiento. Aquí, se postula a que el problema está en el discurso matemático escolar (dME), entendiendo por tal, aquel "paradigma educativo que norma y regula a las matemáticas escolares. Es justamente lo que lleva a los docentes a repetir las mismas clases aun con escasos logros en el aprendizaje de los estudiantes" (CANTORAL, 2013, p. 64). Ante esto, socioepistemología se propone incentivar otro discurso, que rompa la manera en que, actualmente, se abordan los contenidos escolares; en otras palabras, plantea un rediseño del dME, que no esté centrado exclusivamente en

\footnotetext{
1 Entendemos a la matemática funcional como aquella que se incorpora a la vida de las personas para transformarla, que sea parte de su cotidiano.

${ }^{2}$ Concepto que se explicará, con más detalle, en el punto 3 del presente artículo.
} 
conceptos, sino que ofrezca resignificar la matemática, con el propósito de que ésta sea funcional.

Considerando este marco teórico, frente a la situación descrita, postulamos que la introducción del plano cartesiano en estudiantes chilenos de $5^{\circ}$ básico (10 a 11 años de edad) carece de significado y funcionalidad, es decir, se trata y se usa el plano como un saber prescrito, lo cual lleva a que las tareas escolares escasamente exijan su comprensión, hecho que provoca en el aprendiz dificultades para comprender los significados de aquellos tópicos que ahí se ponen en juego.

Las investigaciones de Gascón (2002) y Henríquez (2014) nos reportan la riqueza que posee el plano cartesiano como articulador entre la geometría sintética y la geometría analítica, pero, al mismo tiempo, destacan y evidencian que su enseñanza se limita solo a la algebratización de la geometría.

Atendiendo tales antecedentes, enfrentados a una situación específica, donde el plano cartesiano se pone en juego, nos preguntamos: Los estudiantes ¿tienen la necesidad de construir y usar un plano cartesiano, sin previa caracterización? ¿Qué argumentos señalan los estudiantes cuando recurren a un sistema de referencia? ¿Existen elementos históricoepistemológicos que sean comunes a los utilizados por los estudiantes cuando usan el plano cartesiano?

El objetivo de esta investigación fue resignificar el plano cartesiano a través del diseño de una situación específica, que promueva en un grupo de estudiantes de quinto básico la necesidad de su construcción.

\section{Antecedentes y Problemática}

Durante la enseñanza escolar ${ }^{3}$ se realizan actividades cuyo propósito es que los estudiantes desarrollen la orientación en el plano, la ubicación espacial, la compresión de gráficas y el uso adecuado de los ejes cartesianos (MINEDUC, 2012). Sin embargo, aun cuando en la escuela se proceda con esta clase de tareas, los estudiantes continúan con dificultades, perdido la riqueza que aporta al manejo del espacio bidimensional orientado.

Con respecto a la introducción de los ejes cartesianos, Godino y Ruiz (2002, p. 578) señalan al movimiento de reflexión como facilitador tanto del proceso de localización y ubicación espacial como - al ser un movimiento rígido sobre el plano - de la introducción

${ }^{3}$ El sistema escolar chileno se organiza en un año de educación preescolar obligatoria (kínder), ocho años de enseñanza básica y, luego, cuatro años de enseñanza media. 
oportuna de los sistemas de coordenadas. En cuanto al plano cartesiano como sistema de referencia, ambos autores lo relacionan con la ubicación de puntos en el plano y también con las representaciones cartográficas, planimétricas y de red de coordenadas geográficas.

Por su parte, Bautista (2013) al resignificar la función par e impar, se propuso como objetivo específico resignificar el plano cartesiano a través de las simetrías axial y puntual de diferentes funciones en el plano. Para este propósito, presenta diversas gráficas en el plano para luego, utilizando el software Geogebra, preguntar por los ejes a través de la observación de funciones y simetrías. Los estudiantes juegan con los ejes cartesianos, los manipulan, y tales ejes y el plano se les hacen necesarios para ubicar las figuras (BAUTISTA, 2013).

En nuestra investigación, la reflexión juega un rol clave al introducir el plano cartesiano, pero no facilitaremos los ejes ortogonales, pues se espera que emerjan de la necesidad de su uso y, con ello, la manipulación y la caracterización del plano por parte de los estudiantes.

Miranda, Radford y Guzmán (2007), nos reportan la mirada novicia de los estudiantes sobre el Plano Cartesiano, donde interpretan sus signos de manera idiosincrática o singular. El profesor explica a los estudiantes la noción de distancia para apoyar el proceso de interpretación de la gráfica, aunque una de las estudiantes hace un intento por incorporar el Plano Cartesiano, como un gráfico que permite marcar la posición y dar una explicación del movimiento, la discusión de la relatividad del movimiento se aminora con la discusión de un movimiento descrito sin más detalles (MIRANDA; RADFORD; GUZMAN, 2007, p. 23).

Para efectos de esta investigación, la noción de distancia la hemos considerado como un elemento central para la resignificación del plano cartesiano, en contraste a la investigación de Miranda, Radford y Gusmán (2007), creemos que esta noción debe emerger desde los sujetos en estudio y no de la explicación de un tercero, pues si bien, la intervención del docente permite concienciar sobre los significados culturales, la resignificación permite construir significados propios permeados del contexto, la historia y la cultura.

Años antes, Nemirosky, Tierney y Wright (1998, p.168), indagan en la descripción que realizan estudiantes de quinto grado sobre el movimiento de objetos, observan que las representaciones en gráficos de distancia y velocidad no son inherentes pero, crear enfoques gráficos antes de introducir los convencionales, permite a los estudiantes tomar conciencia de los elementos que necesitan para comunicar sus representaciones.

En nuestro caso, nos interesa pesquisar en las tareas realizadas por los estudiantes aquellos elementos que muestren la construcción de un plano cartesiano, es decir, qué 
argumentos utilizan para comunicar sus representaciones que develen elementos claves para su resignificación.

En las Bases Curriculares chilenas y los textos de estudio, observamos que el plano cartesiano se trabaja como un recurso que provee al profesor la entrada de ciertos contenidos matemáticos y a los estudiantes aprender nociones, tanto de localización y ubicación de puntos, como de comprensión y lectura de gráficas en un sistema de referencia establecido y sin previa construcción. A modo de ejemplo, la primera introducción que se realiza del plano cartesiano, como tal, sugerida en el texto de estudio entregado a los estudiantes de quinto básico y bajo el objetivo de aprendizaje "Identificar y dibujar puntos en el primer cuadrante del plano cartesiano, dadas sus coordenadas en números naturales" (MINEDUC, 2012. p.120), presenta lo siguiente (Figura 1):

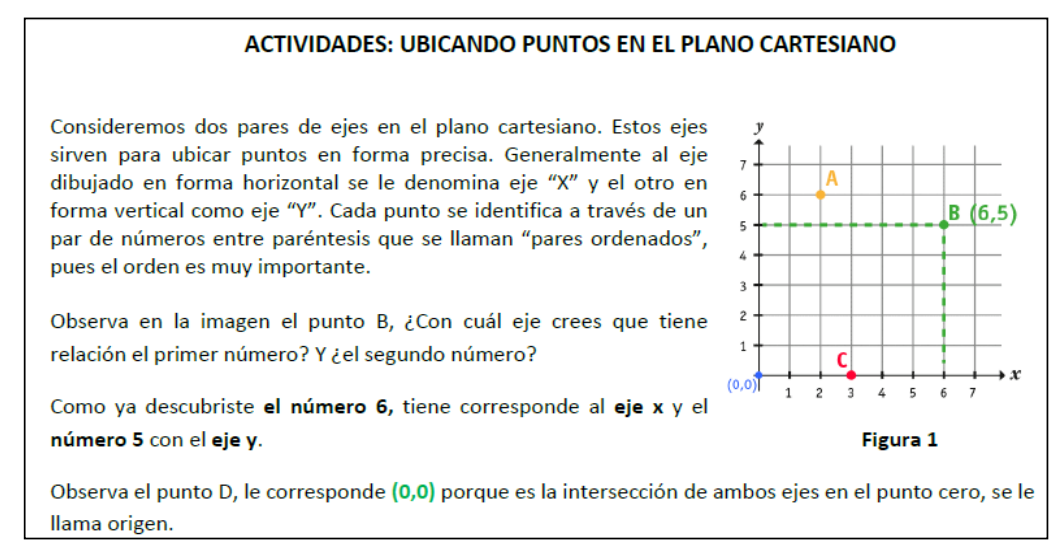

Figura 1 - Introducción del plano cartesiano en $5^{\circ}$ básico Fuente: ASKEY (2014, p. 190).

Respecto al dME, evidenciamos en el texto citado la validación de una enseñanza que introduce los ejes cartesianos como un saber prescrito, donde la capacidad de argumentar y construir conocimiento matemático se ve opacada por la necesidad de transmitir un contenido normado, pero desprovisto de significado. Cordero y Flores (2007, p. 14) indican que el dME "considera a la matemática como un conocimiento acabado y trata los conceptos matemáticos en las acciones de enseñanza como actos repetitivos o de memorización”.

El plano cartesiano no es construido, sino entregado a los estudiantes, con algunas de sus características para ubicar puntos. Se distingue un conocimiento establecido, falta de claridad sobre la construcción de este sistema de referencia donde los niños y niñas puedan comprender su uso, finalmente, los estudiantes deben aprender cómo ubicar puntos siguiendo reglas establecidas.

Por esta razón, postulamos a que una de las dificultades en la comprensión y uso del plano cartesiano se vislumbra en la introducción desprovista de sentido de los ejes 
ortogonales; el dME valida dicha difusión como un saber establecido, que no requiere de una construcción significativa por parte del estudiante.

Esta investigación propone atender, en parte, a esta dificultad, a través de un marco de referencia que provea al dME de otro tipo de situaciones, donde se pueda visualizar significados, procedimientos $\mathrm{y}$ argumentos con el propósito de resignificar el plano cartesiano, invitando al estudiante a ser parte de la construcción de su conocimiento. Para tal efecto, proponemos el diseño de una situación en la cual emerja dicho conocimiento.

De acuerdo a nuestro enfoque socioepistemológico, consideramos elementos histórico- epistemológicos que nos proveen de aspectos a incorporar en el diseño. A continuación, se explicita una breve descripción al respecto.

\subsection{Algunos elementos histórico-epistemológicos sobre el uso del Plano Cartesiano}

El interés por conocer, interpretar y predecir la realidad a través de la razón ha llevado al ser humano a utilizar sistemas de referencias que permiten ubicar y caracterizar elementos u objetos en el plano y el espacio. Estos sistemas de referencia emergen en ciertos escenarios, donde son percibidos, definidos y puestos en uso con el propósito de resolver problemas.

Si bien es cierto, el plano cartesiano se atribuye a René Descartes, existen algunos usos que se realizaron previos a la época del filósofo que dan cuenta de este sistema de referencia, lo cual nos proporciona información valiosa a considerar para el diseño de situación.

Aproximadamente durante el año 350 a.C., en los estudios del matemático griego Menecmo, se observa el uso de ejes ortogonales para dar respuestas a ciertos problemas de ubicación de curvas y puntos en un sector determinado del plano y el espacio. Al cortar un cono con un plano perpendicular a la generatriz, Menecmo observa un par de ejes y, utilizando este sistema de coordenadas, descubre aquellas curvas asociadas a la sección del cono, más adelante conocidas como elipse, parábola e hipérbola, encontrando, por ejemplo, puntos de intersección entre dos parábolas que satisfacen una proporción (BOYER, 1986) (Figura 2).

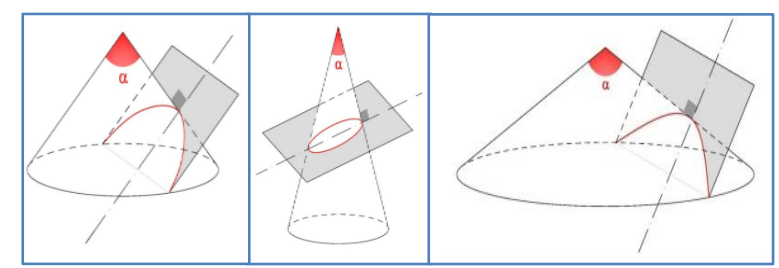

Figura 2 - Plano perpendicular a la generatriz de la cónica Fuente: MUÑOZ (2015, p. 5). 
Posteriormente, los estudios planimétricos de Apolonio sobre las secciones cónicas son un avance en el uso de los ejes, pues abandona la construcción tridimensional de aquellas curvas para realizar el estudio de ellas en el plano. Apolonio considera un sistema de coordenadas reconociendo dos rectas de referencia principales, al observar dos diámetros conjugados que se cortan en un punto (Figura 3).

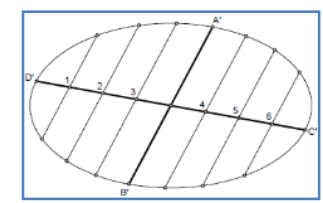

Figura 3 - Diámetros conjugados

Fuente: BARTOLOMÉ (2015).

En el siglo XVII, la matemática de René Descartes estaba ligada a las tradiciones anteriores, llevada a cabo por los griegos. El método cartesiano se funda en las cadenas de razonamiento que utilizan los geómetras para sus demostraciones, entre ellas, “ordenar los conocimientos, empezando siempre por los más sencillos, elevándose por grados hasta llegar a los más compuestos, y suponiendo un orden en aquellos que no lo tenían por naturaleza" (DESCARTES ${ }^{4}, 1637$, p. 17). Al enfrentar un problema, éste se descompone, se organiza y se resuelve. Con esto, Descartes evidencia un método que los matemáticos utilizan para llegar a demostraciones científicas y verdaderas, de lo simple a lo complejo.

Descartes toma éste razonamiento y se propone estudiar aquellas ciencias en que, "siendo diferente su objetivo, coinciden [...]. Por este procedimiento pondría a contribución el análisis geométrico y el álgebra y corregiría los defectos con las ventajas que su uso reportara" (DESCARTES, 1637, p. 18). Los pensamientos que guiaron a Descartes en la creación de sus producciones nos pone de manifiesto el rol del álgebra y de la geometría en ellos. "Descartes propone tomar lo mejor del álgebra y la geometría y corregir los defectos de una con ayuda de la otra" (MORRIS, 1972, p. 408).

En cuanto a la utilización del plano y uso de coordenadas, el filósofo y matemático, considera que "de todas las dimensiones de magnitud continua, la que más distintamente concebimos son la longitud y la anchura. No debemos de fijar la atención en varias a la vez, sino a comparar dos que difieran entre sí” (DESCARTES, 1628, p. 158). Esto adquiere significado al considerar solo dos dimensiones cuando se ubican puntos, curvas, figuras etc., en el plano, utilizando coordenadas rectangulares u oblicuas, siendo estas últimas las usadas por Descartes.

\footnotetext{
${ }^{4}$ Tratado escrito en latín por René Descartes y publicado luego de su muerte, en el año 1701. Se cree que este manuscrito es la base de cada una de sus obras. Extraviado el texto original, solamente queda una traducción realizada en francés y las traducciones al español que de ella se desprenden.
} 
Fermat estudia la ecuación lineal para enfocarse en los lugares planos de Apolonio, y elige un sistema de referencia arbitrario para representar la ecuación. A diferencia de la utilización de coordenadas oblicuas, el uso del sistema que realiza Fermat es semejante al uso actual, utilizando en sus estudios ejes del tipo ortogonal (BOYER, 1986, p. 439).

\subsection{Consideraciones}

El plano cartesiano fue usado como sistema de referencia para el estudio de curvas, representaciones gráficas de una función, construcción de ciertos elementos geométricos, como puntos, o describir el movimiento de objetos. Los ejes emergieron por la necesidad de responder a ciertos problemas matemáticos de la época o retomar discusiones matemáticas para dar posibles soluciones. Desde Menecmo, se utilizó la noción de distancia para describir la posición de cualquier elemento a partir del punto de origen de los ejes.

En relación a Descartes, rescatamos el rol del álgebra, de la geometría; y la necesidad de que el estudiante conozca elementos básicos para construir ideas complejas. En nuestro caso, pretendemos introducir de manera diferente el plano cartesiano en niños de educación básica, donde distingan aspectos geométricos que el dME no explicita. De esta manera, pretendemos resaltar aspectos esenciales que emerjan en los argumentos de los estudiantes y, desde esa vereda, tomar aquellas nociones que permitan su resignificación.

Atendiendo el recorrido histórico, rescatamos tres elementos que consideramos importantes para el diseño de nuestra situación. En primer lugar, el análisis de ciertas figuras y curvas que cambian de posición en el mismo plano, con el propósito que los estudiantes puedan percibir un sistema de referencia. La observación y ubicación de puntos para que emerja la necesidad de construir los ejes, en función de la noción distancia. Finalmente, un momento donde se presente a los participantes un problema a resolver, con el propósito de que construyan un plano cartesiano, introduciendo el concepto de origen como aquel punto de intersección de los ejes.

Entre los aspectos teóricos para dar cuenta de la resignificación del plano cartesiano, ocupamos un marco de referencia reportado en investigaciones de Cordero (2003) y Cordero, Cen y Suarez (2010).

\section{Marco Teórico}


La teoría Socioepistemológica de la Matemática Educativa realiza una crítica al dME (CORDERO et al., 2015), pues este valida la enseñanza de los objetos matemáticos como conocimientos acabados y establecidos, desatendiendo por completo el significado de la construcción social de ese conocimiento. Compartimos con Buendía (2006) que no solo los objetos matemáticos, sino también las prácticas que los generan, son importantes en la adquisición del conocimiento matemático.

Desde la Socioepistemología

[...] se deja de analizar exclusivamente los conceptos matemáticos para empezar a analizarlos conjuntamente con las prácticas que acompañan su producción, [esto es] una descentración del objeto matemático [que] no es su abandono [sino más bien influir en] su valor en uso (CANTORAL, 2013, p. 46).

Así, la Socioepistemología se centra en la funcionalidad de ese conocimiento matemático, en el valor en uso que se pone en juego frente a una situación específica. Éste se logra ante un conocimiento que se construye y reconstruye en la organización del grupo humano, para ello se requieren de marcos de referencia que promueva una matemática funcional en que las situaciones específicas den cuenta de su resignificación y construcción.

Cuando nos referimos a una matemática funcional, hablamos de una en la cual el estudiante adquiera conocimientos, actitudes y habilidades que sean de utilidad para comprender el mundo que lo rodea; es decir, un conocimiento incorporado orgánicamente al ser humano, que transforma su realidad; en oposición al conocimiento utilitario, carente de significados para el estudiante (CORDERO, 2006).

La funcionalidad permite que el conocimiento matemático adquiera significados dentro y fuera del ámbito escolar, lo que conlleva que el conocimiento matemático sea valorado por los estudiantes y no quede al nivel de aplicación de fórmulas que simplemente olvida y que no es capaz de extrapolar a otras situaciones (MORALES; ROSAS, 2016, p. 250).

Al igual que Cantoral (2013, p. 27), consideramos que

Los conceptos y procesos matemáticos que se ponen en funcionamiento en un acto didáctico pueden no ser propiamente objetos matemáticos en el sentido clásico [...], típicamente aceptado por la comunidad matemática o en el ámbito de la noosfera educativa que se expresa en el curriculum oficial, en forma explícita o tácita, sino que se trata de acciones, actividades y prácticas que participan de otros ámbitos de la actividad humana y que, sin embargo, no son reconocidas por el dME.

Por medio de la resignificación, la Socioepistemología se propone indagar en aquellos elementos que permiten caracterizar las condiciones necesarias que se deben poner en juego durante la enseñanza escolar para favorecer la construcción de conocimientos matemáticos. La búsqueda de las condiciones necesarias están arraigadas en la naturaleza misma del 
conocimiento que se desea estudiar (CORDERO; SUÁREZ, 2008). Debido a ello, consideramos el carácter histórico-epistemológico, pues ello implica indagar en el uso que ha tenido y la resignificación se produce a través de ese uso.

Por su parte, Martínez (2005, p. 200) agrega al respecto que la noción de resignificación es un elemento que devela que el conocimiento tiene "significados propios, contextos, historia e intención"; lo que permite enriquecer el significado de los conocimientos al interior de los grupos humanos. De aquello podemos señalar que la resignificación que realice el grupo de estudiantes de un conocimiento matemático no es única o acabada, puede modificarse, pues depende del uso de ese conocimiento frente a una situación específica; cada grupo humano puede establecer diferentes usos según la necesidad de ese conocimiento. Al igual que Morales y Cordero (2014, p. 5) comprenderemos como resignificación a

[...] la construcción del conocimiento mismo en la organización normado por lo institucional; es decir, es el uso del conocimiento en una situación específica donde se debate entre su funcionamiento y forma acorde con lo que organizan los participantes. La resignificación está articulada con los aspectos funcionales y del uso del conocimiento en cuestión [...].

En síntesis, resignificar un conocimiento matemático como un conocimiento en uso implica que los participantes de un grupo logren construir ese conocimiento, prediciendo algo desconocido, consigan colocarlo en funcionamiento otorgándole significados propios. De tal manera, es necesario encontrar elementos para que la situación favorezca la resignificación en aquel grupo de participantes.

Las investigaciones reportadas por Cordero (2003) y Cordero, Cen y Suarez (2010) exhiben una Socioepistemología del Cálculo y el Análisis, la que nos provee de marcos de referencia para atender nuestro propósito. Estas investigaciones utilizan situaciones de variación, de transformación y de aproximación para atender temas matemáticos desde otra perspectiva, diferente a la entregada por el dME habitual.

Es por ello, que hemos considerado aspectos de una situación de variación y de transformación para poner en juego, teniendo presente, además, tanto los aspectos históricoepistemológicos, como los antecedentes recopilados que den cuenta de la resignificación del plano cartesiano. De esta manera, nuestras situaciones tendrán significados, procedimientos y argumentos que emergen a partir del trabajo realizado por los mismos estudiantes.

\section{Aspectos Metodológicos}


La metodología empleada está bajo el paradigma cualitativo (HERNÁNDEZ; FERNÁNDEZ; BAPTISTA, 2010), pues indagamos en el proceso de construcción de un determinado conocimiento matemático, develando en los argumentos e interacciones de los participantes, aquellos elementos comunes que permiten resignificar el plano cartesiano.

Para la elaboración del diseño de situación, consideramos elementos históricoepistemológicos pertinentes con el objeto matemático de estudio, como también elementos teóricos, dado que la propuesta tiene la intencionalidad de proveer un contexto en que los participantes generen significados, procedimientos, argumentos y nociones matemáticas que puedan develar la construcción del plano cartesiano.

Para el análisis de los resultados obtenidos consideramos la Ingeniería Didáctica (ARTIGUE, 1995), pues permite confrontar los análisis a priori y a posteriori de la situación. Las variables de control serán evidenciadas de acuerdo a la estructura que se presenta en el diseño, pues cada momento está intencionado de acuerdo a los elementos recogidos en los antecedentes, en los aspectos históricos-epistemológicos y en el marco teórico adoptado.

\subsection{Contexto y recogida de datos}

Considerando una de las preguntas que provocó esta investigación - Los estudiantes ¿tienen la necesidad de construir y usar un plano cartesiano, sin previa caracterización? -, hemos seleccionado sujetos de investigación que cursan $5^{\circ}$ básico, pues en este nivel se presenta explícitamente desde las Bases Curriculares (MINEDUC, 2012) y por vez primera, el contenido plano cartesiano.

La situación se lleva a cabo en una clase experimental, con un grupo de seis estudiantes, seleccionados de forma aleatoria. Estos participantes aún no han trabajado con el plano cartesiano como tal, pues queremos promover un diseño de situación que permita construir dicho conocimiento a través del uso que realicen los estudiantes, dejando de lado la presentación habitual promovida y replicada por el dME.

Los estudiantes pertenecen a una escuela cuyo programa de estudio se basa en la propuesta por las Bases Curriculares (MINEDUC, 2012), por tanto, el programa de estudio de esta institución escolar tiene como escenario el dME imperante en el sistema educativo actual. Cabe resaltar que ninguna de las investigadoras conocía al grupo de estudiantes, por tanto, esta selección no consideró las características personales de cada uno, solamente que cursaran el mismo nivel. 
La recogida de datos se obtuvo a través de la implementación del diseño de situación con las respuestas de los estudiantes; también incluyó entrevistas semiestructuradas, es decir, se planificó el diseño de preguntas abiertas que son claves y que deben estar presentes en la situación con el propósito de provocar la predicción, la argumentación, la discusión y la construcción de conocimiento de acuerdo a la interacción generada por el grupo de participantes.

También se pensó en la realización de preguntas que surgieran de manera espontánea en el desarrollo de la situación, con el propósito de profundizar en las respuestas y argumentos emitidos por uno o varios de los participantes. Además, en cada momento, se realizaron grabaciones para obtener el registro audiovisual de las argumentaciones de los estudiantes, las cuales fueron previamente informadas y aceptadas, por los participantes y por el equipo directivo de la escuela.

\subsection{Diseño de Situación}

Se diseñaron dos situaciones, cada una de ellas contiene dos momentos específicos, y un tercer momento que unifica ambas situaciones. Las situaciones 1 y 2 están elaboradas considerando los elementos declarados en los puntos 2 y 2.2. El momento que unifica ambas situaciones está pensado para que el estudiante construya y resignifique el plano cartesiano y, con ello, aportar al rediseño del discurso matemático escolar.

En la situación 1 participaron tres estudiantes, en la situación 2 participaron otros tres; en el tercer momento, los seis integrantes quedaron reunidos para discutir y finalizar la actividad. La siguiente imagen ejemplifica la estructura del diseño; en ella se menciona lo esencial de cada momento (Figura 4).

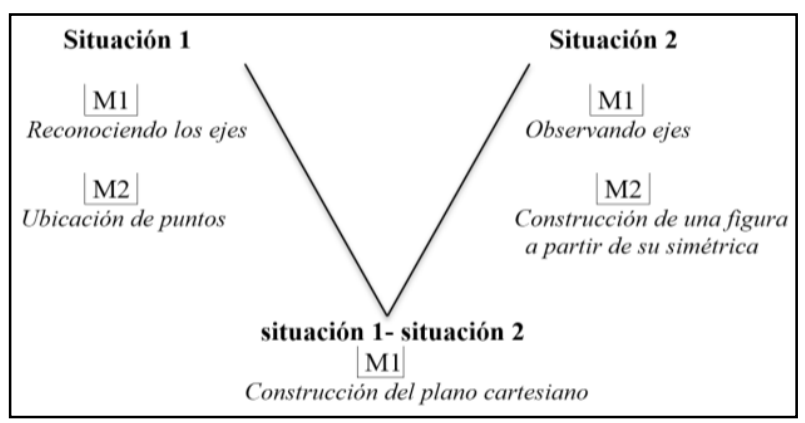

Figura 4 - Estructura del diseño con sus momentos Fuente: Elaboración Propia.

A continuación, se presenta cada situación con sus respectivos momentos en función del análisis a priori realizado. 


\subsubsection{Situación 1}

\subsubsection{Momento 1: Reconociendo los ejes}

Este momento tuvo por objetivo que los estudiantes percibieran los ejes del plano cartesiano. Se mostró a un grupo de tres estudiantes cinco actividades en el software Geogebra, cuya finalidad y análisis a priori fue la predicción, observación, descripción, argumentación y experimentación con gráficas y sus simétricas, las cuales varían a través del uso de botones con el fin de provocar el reconocimiento de los ejes.

Los tres estudiantes observaron y manipularon el software en cinco exploraciones; en cada una de estas se presentaron figuras con sus simétricas respectivas, de modo que los participantes, al deslizar unos botones, observaran que ambas figuras se movían de forma horizontal o vertical, en igual dirección pero distinto sentido, como si entre ambas estuviese la presencia de un espejo.

\subsubsection{Momento 2: Justifico la ubicación de puntos}

Los estudiantes experimentan con material concreto (lápiz y papel). Se espera que los participantes representen de manera gráfica la presencia de un sistema de referencia.

Luego de explorar con el software, se presentó al grupo ciertas figuras con sus respectivas simétricas. En una de esas figuras se ubica un punto y se le solicita a los estudiantes que señalen dónde ubicarían ese punto en la figura simétrica, indicando al menos tres argumentos de su decisión. Los estudiantes discutieron y respondieron a dicha tarea.

Se solicitan al menos tres argumentos, porque uno de ellos puede surgir al doblar el papel, otro, basarse en la visualización; sin embargo, esperamos que un tercer argumento sea la noción de distancia para argumentar la posición de los puntos con respecto a los ejes.

En este momento, los estudiantes pueden doblar el papel, solicitar regla para situar los ejes y medir la distancia de los ejes con respecto al punto para ubicar el punto simétrico.

Además, una de las exploraciones permite que los participantes tengan la posibilidad de percibir más de dos ejes de referencia, pues la imagen cuenta con una figura en donde podemos observar su reflejo en forma vertical y horizontal.

\subsubsection{Situación 2}




\subsubsection{Momento 1: Observo ejes}

En este momento, los estudiantes manipulan fichas con figuras geométricas. Se presentó a un grupo de tres estudiantes una serie de fichas con gráficas y curvas de colores que tenían reflexión horizontal, vertical e inclinada; aquí se esperaba que los participantes describieran cómo se construye una figura a partir de la otra. Como primera etapa de la construcción de las coordenadas, el objetivo de la situación es que surja la necesidad de argumentar con los ejes que no se encuentran explícitos en las fichas.

Las figuras y las curvas presentes en esta situación tenían colores, puesto que es un elemento al cual podíamos recurrir para solicitar a los estudiantes la construcción de una figura simétrica. A través del color, los participantes pueden identificar la ubicación de la figura simétrica que se les solicita en el segundo momento.

\subsubsection{Momento 2: Construcción de una figura a partir de su simétrica}

Luego del primer momento y de percibir la presencia de los ejes, los estudiantes deben dibujar las simétricas respectivas de figuras y curvas. Aquí se espera que los participantes argumentasen utilizando el concepto de distancia. En este momento, los estudiantes proceden a construir la simétrica de una figura utilizando la noción de distancia con respecto al eje para justificar la posición. Los participantes, para trazar un eje, pueden solicitar regla y medir la distancia de la figura con respecto al eje. Posteriormente, debían replicar la medida de esta distancia al otro lado del eje para dibujar la figura simétrica.

\subsubsection{Situación 1 - Situación 2}

\subsubsection{Momento 1: Construcción del plano cartesiano}

Se diseñó un momento que unificara ambas situaciones, reuniendo al grupo de seis estudiantes pues, a partir de sus distintas experiencias previas, enriquecerían la discusión. Quienes trabajaron con Geogebra, grupo 1, pueden predecir y discutir con sus compañeros en torno a las características de los ejes solicitados, mientras que el grupo 2 puede aportar desde la manipulación con instrumentos de medición, la construcción que realizaron de las figuras simétricas y el concepto de distancia para ubicar figuras y puntos en el plano. 
De esta manera, utilizando una figura situada en el centro de un disco hecho de papel, negro por un lado y blanco por el lado de trabajo, cada uno de los estudiantes que participó en las situaciones previas tuvo que construir dos figuras simétricas, utilizando ejes distintos, es decir, cada integrante debía construir dos ejes de referencia distintos entre sí y, además, no debían diseñar un eje de referencia igual al de otro compañero. Luego, en grupo, determinarían cuál es la mejor construcción.

Con esta tarea no promovemos el uso estático de los ejes y se espera que emerjan varios ejes, entre ellos los ejes ortogonales que forman el plano cartesiano.

No se utilizó una hoja rectangular, porque los lados de la hoja podrían ayudar a fijar los ejes paralelos a los lados. En cuanto a los colores del disco de papel, postulamos que los estudiantes doblarían el disco para ubicar tanto los ejes como la figura simétrica; si el papel es negro por uno de los lados, evitará que los estudiantes puedan copiar la figura por medio del reflejo del papel, contribuyendo a la búsqueda de una nueva estrategia.

\section{Resultados}

El siguiente apartado muestra parte del análisis a posteriori, se presenta un extracto de los resultados obtenidos e imágenes de las producciones de los participantes que evidencian el logro del objetivo propuesto en esta investigación. Se exponen ejemplos de cada momento, cada uno de ellos da cuenta del proceso de resignificación del plano cartesiano, en que podemos identificar que los estudiantes percibieron y construyeron los ejes, utilizaron la noción de distancia con respecto a los ejes para argumentar la posición de un punto o figura en el plano, lograron establecer coordenadas rectangulares, y argumentaron la posición del punto de intersección de los ejes ortogonales como el punto de origen o de partida del sistema de referencia. Todo esto fue capturado con sus significados, procedimientos y argumentos en cada tarea planteada.

\subsection{Observación de los ejes}

En el momento 1 de ambas situaciones los estudiantes percibieron la presencia de ejes, señalando la existencia de un espejo; mencionaron la presencia de figuras iguales. Los participantes demarcaron los ejes y argumentaron la presencia de aquellos (Figura 5). 
Profesor: ¿Qué observan?

Estudiante 1: Es como un espejo

Profesor: ¿Dónde ubicarían este espejo?

Estudiante 2: (muestra según se ve en imagen

1)

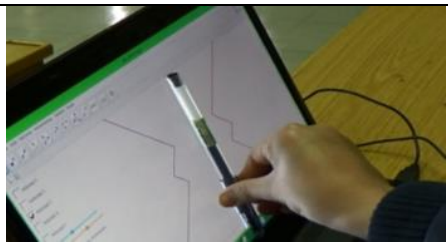

Figura 5 - Situación 1 - Momento 1 - Exploración 2

Fuente: ARAVENA (2015).

En la Figura 6 tenemos la Situación 2 - Momento 1:

Profesor: A partir de la figura roja ¿Cómo

construirías la figura morada?

Estudiante 4: Son como espejos.

¿Construyo un espejo?

Profesor: ¿Dónde construirías ese espejo

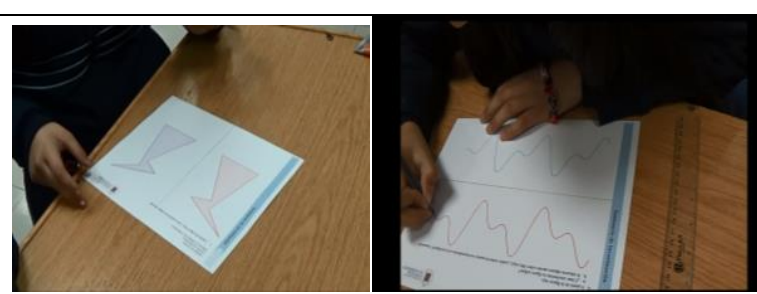

(línea central trazada por el grupo)

\section{Figura 6 - Situación 2 - Momento 1} Fuente: ARAVENA (2015).

En ambos momentos de estas situaciones los participantes argumentan la existencia de los ejes, señalando que son como espejos. Los estudiantes discuten y señalan la existencia del reflejo de una figura con respecto a la otra. Sin embargo, en la exploración 5 de la situación 1 , los participantes logran observar que estos reflejos se pueden traslapar (Figura 7).

Profesor: ¿Dónde ubicarían el espejo?

Estudiante 2, muestra el consenso del grupo.

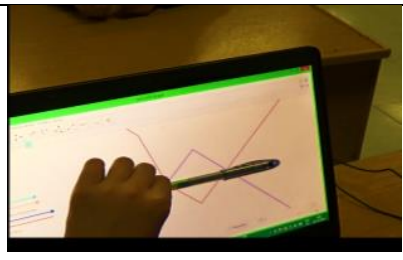

Figura 7 - Situación 1 - Momento 1 - Exploración 5

Fuente: ARAVENA (2015).

Por otro lado, al deslizar los botones del software encuentran una imagen donde argumentan que es posible poner el espejo de forma horizontal y vertical (Figura 8).

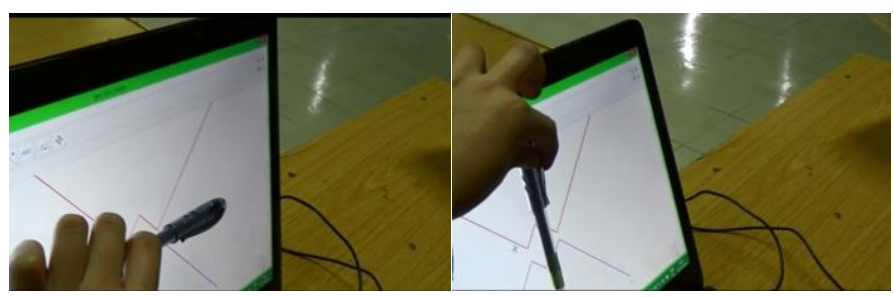

Figura 8 - Situación 1 - Momento 1 - Exploración 5 Fuente: ARAVENA (2015).

\subsection{Construcción de los ejes y uso de distancia para ubicar puntos y figuras}


En el momento 2 de cada una de las situaciones, los participantes recurrieron a la construcción de los ejes, y solicitaron regla para medir las distancias. Los estudiantes ubicaron el eje y midieron con regla la distancia de los puntos al eje para justificar la construcción de otros puntos y/o figuras (Figura 9).

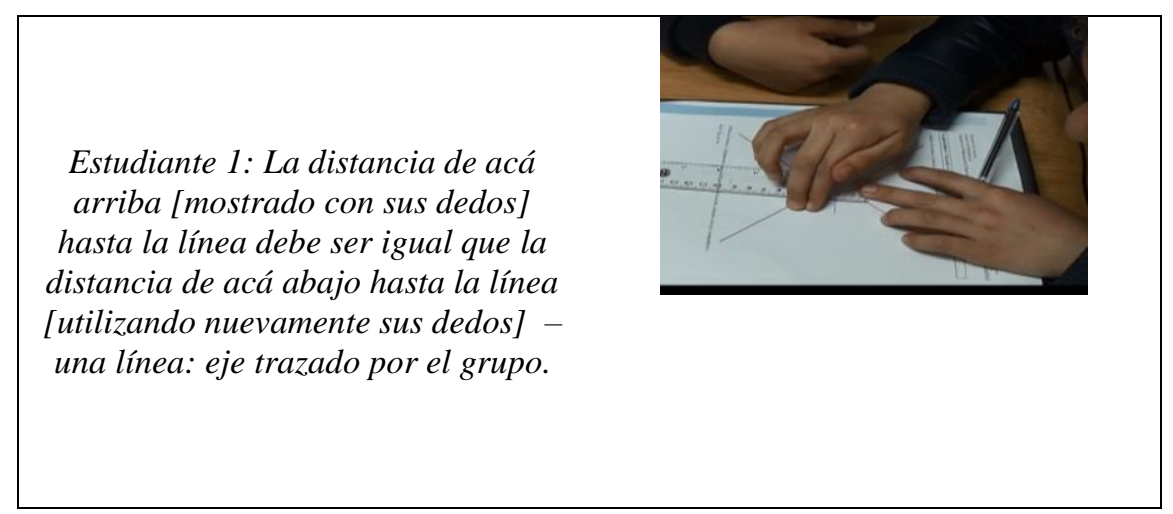

Figura 9 - Situación 1 - Momento 2 - Exploración 6 Fuente: ARAVENA (2015).

Con la exploración 7, los estudiantes que participaron de la situación 1 construyeron ejes ortogonales y justificaron la ubicación de los puntos según la distancia existente con respecto a los ejes (Figura 10).

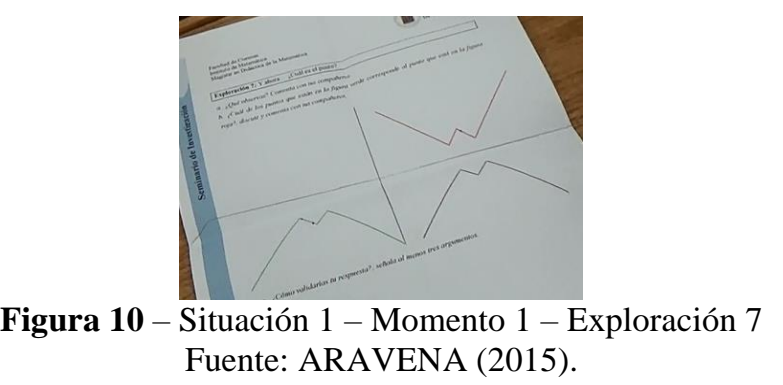

Los participantes no solo construyeron ejes ortogonales, pues con una de las exploraciones construyeron distintos ejes, argumentando nuevamente con la distancia la ubicación de los puntos con respecto a los ejes (Figura 11).

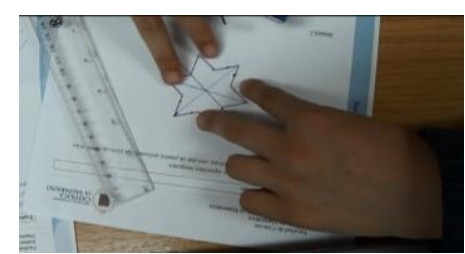

Figura 11 - Situación 1 - Momento 1 - Exploración 8 Fuente: ARAVENA (2015).

En cuanto al momento 2 de la situación 2, los participantes construyen una figura a partir de otra. Si bien es cierto, se presenta un segmento que deben utilizar como eje, los participantes doblan la hoja para prolongar este segmento y con la regla miden la distancia de la figura original con respecto al eje, para luego replicar esta distancia al lado opuesto y dibujar la figura simétrica correspondiente (Figura 12). Además, marcan los vértices de la 
figura original como puntos de referencia para ubicar sus simétricos, luego con aquellos puntos simétricos dibujan la nueva figura.

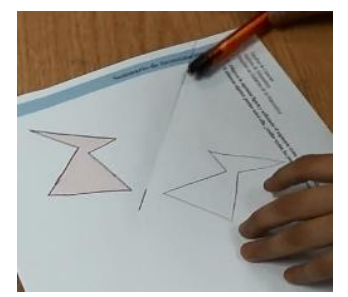

Figura 12 - Situación 2 - Momento 2 - Exploración 7 Fuente: ARAVENA (2015).

\subsection{Construcción del plano cartesiano}

Se reúnen ambos grupos de trabajo y se entrega la siguiente instrucción:

Profesor: cada uno de ustedes debe construir dos espejos [así llamaron a los ejes en los momentos previos]; sin embargo, se deben poner de acuerdo, pues estos espejos no se pueden repetir. Dibujan el espejo y la figura correspondiente a ese espejo.

Los estudiantes discutieron y se apoyaron para construir todos los espejos. En las producciones de los participantes se apreciaron distintos tipos de ejes; los estudiantes que participaron de la situación 1 mostraron a sus compañeros la construcción de una figura sobre otra, cuando el eje se encuentra sobre la figura inicial, y los participantes de la situación 2 enfatizaban constantemente sobre la noción de distancia con respecto a los ejes para dibujar correctamente la figura (Figura 13).

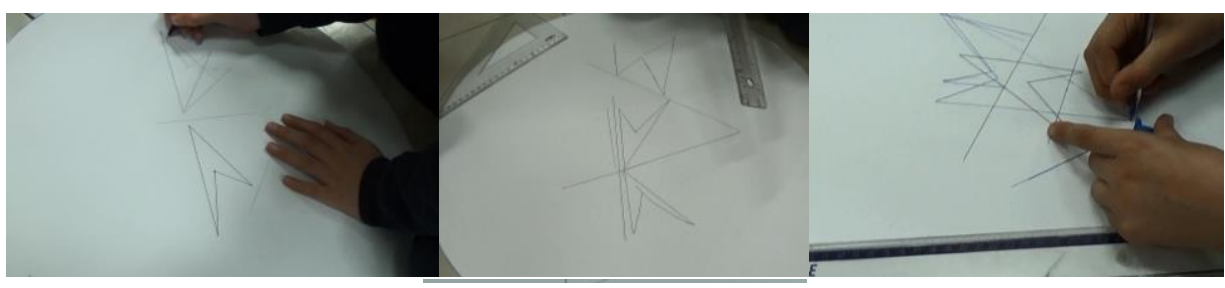

Figura 13 - Situación 1 - Situación 2 - Momento 1

Fuente: ARAVENA (2015).

Luego de construir distintos ejes, uno de los participantes dijo al grupo que sus espejos formarían una cruz. Otro de los estudiantes ayuda en esa construcción. Esta producción fue catalogada por los mismos compañeros(as) como una de las mejores (Figura 14):

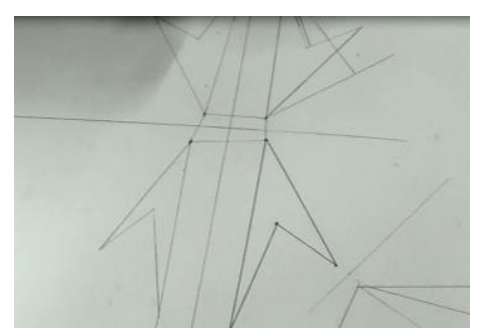

Figura 14 - Situación 1 - Situación 2 - Momento 3 Fuente: ARAVENA (2015). 
Si bien es cierto (que) el grupo señaló que las distancias no estaban del todo bien, procedieron a remarcar ciertos puntos, utilizando coordenadas rectangulares para indicar cuáles eran los simétricos, y señalaron que la distancia de estos puntos con respecto a los dos ejes debían ser las mismas. Finalmente, el profesor pregunta: Si tuviesen que marcar o construir un punto en este plano ¿Dónde lo harían y por qué?

El grupo de inmediato señaló el punto donde se intersectan los dos ejes (Figura 15), al preguntar el por qué, el diálogo sostenido por el grupo fue el siguiente:
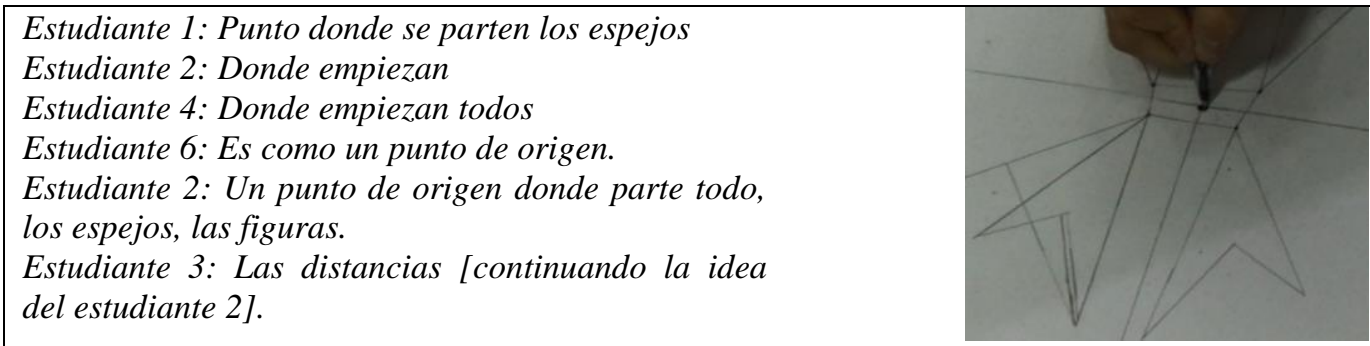

Figura 15 - Situación 1 - Situación 2 - Momento 3 Fuente: ARAVENA (2015).

\section{Conclusiones}

Al confrontar el análisis a priori y a posteriori, observamos que el uso del software facilitó que los estudiantes percibieran la presencia de los ejes en las gráficas; solicitar tres argumentos cuando trabajaron con lápiz y papel favoreció que emergiera la noción de distancia con respecto a los ejes. El momento que unifica ambas situaciones contribuyó a la construcción de distintos ejes y develó aquellos elementos básicos a utilizar en la resignificación del plano cartesiano.

Con la situación propuesta, los estudiantes lograron construir un plano cartesiano sin previa caracterización; más bien las restricciones y el cómo se debía realizar emergió de los diferentes momentos basados en la discusión propia del trabajo llevado a cabo por el grupo. Para referirse a un sistema de referencia, los estudiantes recurren a la noción de distancia con respecto a un eje (el referente) y utilizan coordenadas rectangulares para dicho efecto, pues estas permiten posicionar, a través del largo y del ancho, un punto sobre el plano.

En cuanto a los elementos histórico-epistemológicos comunes a los utilizados por los estudiantes, apreciamos que son capaces de percibir la presencia de los ejes, establecen una relación entre la distancia y la ubicación de un punto o una figura en el plano con respecto a 
los ejes; al igual que Descartes, utilizan coordenadas donde visualizan dos dimensiones, observando un largo y un ancho para situar algo sobre el plano.

Si bien, emergen distintos tipos de ejes, los estudiantes mencionan que la construcción ortogonal es la mejor pues, de acuerdo a los argumentos de los participantes, la visualización de las distancias de una figura o de puntos con respecto al eje, es fácilmente observable si se trabaja con coordenadas rectangulares. Finalmente, emerge el concepto de punto de origen, como el punto más importante del plano, puesto que es el punto desde donde parte todo.

Con este diseño, evidenciamos que los estudiantes de quinto grado construyen ejes de referencia y utilizan coordenadas rectangulares para ubicar y posicionar puntos, figuras geométricas y curvas en el plano. La diferencia de esta situación con el actual dME fue que la construcción de conocimiento se produce al interior del grupo de estudiantes, donde recurren a la noción de distancia, eje de simetría y punto de origen para argumentar parte de sus acciones. Con esto, damos cuenta que existe la posibilidad de provocar en los estudiantes la construcción de un conocimiento, a través de otro tipo de situaciones, distintas a las establecidas por el actual dME.

Desde la postura teórica adoptada, y considerando elementos históricoepistemológicos, este diseño podría ser un prólogo para la resignificación del plano cartesiano como sistema de referencia en diversos contextos. Una de las proyecciones a corto plazo que se visualizan de este trabajo es observar el significado que tiene para los estudiantes las coordenadas en el plano cartesiano y el por qué el uso de coordenadas rectangulares es considerada una mejor opción frente a otros tipos de coordenadas, ya sea oblicua o de otras características.

\section{Referencias}

ACUÑA, C. Concepciones en Graficación, el Orden entre las Coordenadas de los puntos del Plano Cartesiano. Revista Latinoamericana de Investigación en Matemática Educativa, Distrito Federal, v. 4, n. 3, p. 203 - 217, 2001.

ARAVENA, A. Resignificación del Plano Cartesiano: un enfoque socioepistemológico. 2015. Pp 131. Tesis (Magister en Didáctica de la Matemática - Facultad de Ciencia, Instituto de Matemáticas, Pontificia Universidad Católica de Valparaíso, Valparaíso, 2015.

ARTIGUE, M. Ingeniería Didáctica. En: ARTIGUE, M; DOUADY, R.; MORENO, L. Ingeniería Didáctica en Educación Matemática: Un Esquema para la Investigación y la Innovación en la Enseñanza y el Aprendizaje de las Matemáticas. Bogotá, Colombia: Grupo Editorial Iberoamericana, 1995. p. 33-59.

ASKEY, R. (Dir.). Texto del Estudiante, Matemática $5^{\circ}$ básico. Edición especial para el Ministerio de Educación. Chile: Ediciones Galileo, 2014. 
BARTOLOMÉ. Curvas Cónicas: La Elipse. 2015. Disponible en

http://www.dibujotecnico.com/curvas-conicas-la-elipse/ Acceso el 12 de agosto 2015.

BAUTISTA, L. El Rol De La Argumentación Gráfica en la Construcción de Conocimiento Matemático Escolar: El caso de la Paridad e Imparidad de las Funciones. 2013. Pp. 64. Tesis (Doctorado en Didáctica de la Matemática) - Facultad de Ciencia, Instituto de Matemáticas, Pontificia Universidad Católica de Valparaíso, Valparaíso, 2013.

BOYER, C. Historia de la Matemática. Madrid: Editorial Alianza, 1986.

BUENDÍA, G. Una Socioepistemología del Aspecto Periódico de las Funciones. Revista Latinoamericana de Investigación en Matemática Educativa, Distrito Federal, v. 9, n. 2, p. 227 $251,2006$.

CANTORAL, R. Teoría Socioepistemológica de la Matemática Educativa. Barcelona: Editorial Gedisa, 2013.

CORDERO, F. Lo social en el conocimiento matemático: los argumentos y la reconstrucción de significados. Acta Latinoamericana de Matemática Educativa, Clame, v. 16, n. 1, p. 73-78, 2003.

CORDERO, F. La modellazione e la rappresentazione grafica nell'insegnamento-apprendimento della matemática. Revista La Matemática e la sua Didattica, Departamento de Matemática de la Universidad de Bologna, Italia, v. 20, n. 1, p. 59-79, 2006.

CORDERO, F.; FLORES, R. El uso de las gráficas en el discurso matemático escolar. Un estudio socioepistemológico en el nivel básico a través de los libros de texto. Revista Latinoamericana de Investigación en Matemática Educativa, Ciudad de México, México, v. 10, n. 1, p. 7-38, 2007.

CORDERO, F.; SUÁREZ, L. Elementos teóricos para estudiar el uso de las gráficas en la modelación del cambio y de la variación en un ambiente tecnológico. Revista Electrónica de Investigación en Educación de las Ciencias, Centro Argentino de Información Científica y Tecnológica, v. 3, n.1, p. 51-58, 2008. Disponible en: <http://www.scielo.org.ar/scielo.php?script=sci_arttext\&pid=S185066662008000100005>. Acceso el 12 de enero 2015.

CORDERO, F.; CEN, C.; SUÁREZ, L. Los funcionamientos y formas de las gráfica en los libros de texto: Una práctica institucional en el bachillerato. Revista Latinoamericana de Investigación en Matemática Educativa, Ciudad de México, México, v. 13, n. 2, p. 187-214, 2010.

CORDERO, F. et al. El discurso matemático escolar: la adherencia, la exclusión y la opacidad. Barcelona: Editorial Gedisa, 2015.

DESCARTES, R. Discurso del Método, 1637 - Reglas para la Dirección del Espíritu, 1628. Versión Española de Manuel Machado. En: LARROYO, F. Descartes, Discurso del Método, Meditaciones Metafísicas, Reglas para la Dirección del Espíritu, Principios de la Filosofía. México: Editorial Porrúa, 2014. p. 105-169.

GASCÓN, J. Geometría Sintética en la ESO y Analítica en el Bachillerato. ¿Dos mundos completamente separados? Revista Suma, Catalunya, España, v. 39, p. 13-25, 2002.

GODINO, J. Y; RUIZ, F. Geometría y su Didáctica para Maestros, 2002. Disponible en: <http://www.ugr.es/ jgodino/edumat-maestros/manual/4_Geometria.pdf >. Acceso el 12 de enero 2015

HERNÁNDEZ, R.; FERNÁNDEZ, C.; BAPTISTA, P. Metodología de investigación. México: Editorial McGraw-Hill, 2010. 
HENRÍQUEZ, C. El Trabajo Geométrico de Profesores en el Tránsito de la Geometría Sintética a la Analítica en el Nivel Secundario. 2014. Pp. 251. Tesis (Doctorado en Didáctica de la Matemática) - Facultad de Ciencia, Instituto de Matemáticas, Pontificia Universidad Católica de Valparaíso, Valparaíso, 2014.

MARTÍNEZ, G. Los procesos de convención matemática como generadores de conocimiento. Revista Latinoamericana de Investigación en Matemática Educativa, Distrito Federal, v. 8, n. 2, p. 195 $218,2005$.

MINEDUC. Bases Curriculares Educación Básica, 2012. Disponible en:

$<$ http://www.curriculumenlineamineduc.cl/605/articles-21321_programa.pdf $>$. Acceso el 5 de noviembre 2015

MIRANDA, I.; RADFORD, L.; GUZMÁN, J. Interpretación de gráficas cartesianas sobre el movimiento desde el punto de vista de la teoría de la objetivación. Revista Educación Matemática, México, v. 19, n. 3, p. 5-30, 2007.

MORALES, A.; ROSAS, L. Una propuesta para el desarrollo de modelos geométricos en las Educadoras de Párvulos. El caso del polígono. Revista Estudios Pedagógicos, Universidad Austral, Chile, v. 42, n. 2, p. 247-267, 2016.

MORALES, A.; CORDERO, F. La Graficación-Modelación y la Serie de Taylor. Una Socioepistemología del Cálculo. Revista Latinoamericana de Investigación en Matemática Educativa, Ciudad de México, México, v. 17, n. 3, p. 319-345, 2014

MORRIS, K. El pensamiento matemático de la antigüedad a nuestros días. España: Alianza Editorial, 1972. p. 401-450.

MUÑOZ, A. Curvas cónicas desde su origen hasta sus aplicaciones en la actualidad. 2015. Pp 99. Tesis (Máster Universitario de Profesorado en Educación Secundaria Obligatoria, Bachillerato, Formación Profesional y Enseñanza de Idiomas (Especialidad de Matemáticas)). Universidad de Valladoli, España, 2015.

NEMIROSKY, R.; TIERNEY, C.; WRIGHT, T. Body Motin and Graphing. Cognition and Instruction, Taylor \& Francis, Ltd., v. 16, n. 2, p. 119-172, 1998.

SAIZ, I. La Derecha... ¿De quién? Ubicación Espacial en el Nivel Inicial y el Primer Ciclo de la E. G. B. En Panizza, M. (Comp.). Enseñar Matemática en el Nivel Inicial y primer ciclo de la EGB. Buenos Aires: Paidós, 2003. p. 245-287. 